\title{
Synergistic cytotoxic effects of inorganic phosphate and chemotherapeutic drugs on human osteosarcoma cells
}

\author{
ANNAMARIA SPINA, LUCA SORVILLO, EMILIO CHIOSI, ANTONIETTA ESPOSITO, \\ FRANCESCA DI MAIOLO, LUIGI SAPIO, MICHELE CARAGLIA and SILVIO NAVIGLIO \\ Department of Biochemistry, Biophysics and General Pathology, \\ Second University of Naples, Medical School, I-80138 Naples, Italy
}

Received November 16, 2012; Accepted December 11, 2012

DOI: $10.3892 /$ or.2013.2306

\begin{abstract}
Novel therapeutic approaches are required for the treatment of osteosarcoma. Combination chemotherapy is receiving increased attention in order to identify compounds that may increase the therapeutic index of clinical anticancer drugs. In this regard, naturally occurring molecules with antitumor activity and with limited toxicity to normal tissues have been suggested as possible candidates for investigation of their synergistic efficacy in combination with antineoplastic drugs. Inorganic phosphate (Pi) is an essential nutrient for living organisms. Relevantly, Pi has emerged as an important signaling molecule capable of modulating multiple cellular functions by altering signal transduction pathways, gene expression and protein abundance in many cell types. Previously, we showed that $\mathrm{Pi}$ inhibits proliferation and aggressiveness of U2OS human osteosarcoma cells and that $\mathrm{Pi}$ is capable of inducing sensitization of osteosarcoma cells to doxorubicin in a p53-dependent manner. In this study, we extended the role of $\mathrm{Pi}$ in the chemosensitivity of osteosarcoma cells to other anticancer drugs. Specifically, we report and compare the antiproliferative effects of a combination between Pi and doxorubicin, Taxol ${ }^{\circledR}$ and 5-fluorouracil (5-FU) treatments. We found that $\mathrm{Pi}$ increases the antiproliferative response to both Taxol and doxorubicin to a similar extent. On the other hand, Pi did not potentiate the anticancer effects induced by $5-\mathrm{FU}$. These effects were paralleled by apoptosis induction and were cell cycle-dependent. The clinical significance of our data and their potential therapeutic applications for improving osteosarcoma treatment are discussed.
\end{abstract}

Correspondence to: Professor Michele Caraglia or Dr Silvio Naviglio, Department of Biochemistry, Biophysics and General Pathology, Second University of Naples, Medical School, Via L. De Crecchio 7, I-80138 Naples, Italy

E-mail: michele.caraglia@unina2.it

E-mail: silvio.naviglio@unina2.it

Key words: osteosarcoma cells, inorganic phosphate, cytotoxicity, chemosensitivity, combination antitumor therapy

\section{Introduction}

Osteosarcoma is the most common primary malignant tumor of the bone, occurring most frequently in children and adolescents (1). Surgery, radiotherapy and high-dose chemotherapy [with agents such as doxorubicin, methotrexate, cisplatin, 5-fluorouracil (5-FU), Taxol ${ }^{\circledR}$ and etoposide] are mainly effective in patients with localized disease and have improved patient overall survival over the last several years (2).

However, clinically evident metastatic disease is present in $10-20 \%$ of patients at diagnosis. Despite aggressive treatment, more than one third of patients develop recurrent high-grade osteosarcomas, with metastatic disease typically affecting the lung, liver and bone itself, so that the 5-year survival rates are still not greater than $60 \%$. The frequent acquisition of drug-resistant phenotypes and occurrence of secondary malignancies associated with chemotherapy remain serious problems (3). Moreover, the toxic effects of chemotherapy still remain a major drawback in the treatment of osteosarcoma patients. Thus, there is a pressing need for the development of new and alternative approaches to the treatment of osteosarcoma (4).

Combination chemotherapy has received increased attention in order to identify compounds that may increase the therapeutic index of clinical anticancer drugs (5). In this regard, dietary supplements, phytotherapeutic agents and naturally occurring molecules (such as silibinin, resveratrol, plumbagin, benzyl isothiocyanate, 2-methoxyestradiol, DDTD) with antitumor activity and with low toxicity to normal tissues have been suggested as possible candidates for investigation of their synergistic efficacy in combination with antineoplastic drugs (6-11).

Inorganic phosphate $(\mathrm{Pi})$ is an essential nutrient for living organisms. It plays a key role in diverse physiological functions, including osteoblast differentiation and skeletal mineralization (12). Serum Pi level is maintained within a narrow range through a complex interplay between intestinal absorption, exchange with intracellular and bone storage pools, and renal tubular reabsorption and depends mainly on the activity of $\mathrm{Na} / \mathrm{Pi}$ cotransporters (13). $\mathrm{Pi}$ is abundant in the diet, and intestinal absorption of $\mathrm{Pi}$ is efficient and minimally regulated. The kidney is a major regulator of $\mathrm{Pi}$ homeostasis and can increase or decrease its Pi reabsorptive capacity to 
accommodate Pi need. Adequate control of Pi homeostasis is crucial, as a moderate increase in serum Pi concentration and polymorphisms in genes involved in Pi metabolism may result in bone impairment and influence the ageing process and lifespan (14). Relevantly, Pi is emerging as an important signaling molecule capable of modulating multiple cellular functions by altering signal transduction pathways, gene expression and protein abundance in many cell types (15).

Previously, we provided evidence that $\mathrm{Pi}$ inhibits proliferation and aggressiveness of human osteosarcoma U2OS cells identifying adenylate cyclase, $\beta 3$ integrin, Rap1, ERK1/2 as proteins whose expression and function are relevantly affected in response to $\mathrm{Pi}(16,17)$. More recently, we described that in wild-type p53-containing osteosarcoma U2OS cells, and not in p53-null Saos and p53 mutant MG63 osteosarcoma cells, $\mathrm{Pi}$ is capable of inducing sensitization to doxorubicin (18). We provided evidence that the enhancement of doxorubicin-induced cytotoxicity by $\mathrm{Pi}$ occurs via p53-dependent apoptosis and through a mechanism involving ERK1/2 downregulation (18).

Herein, we extended the study of the role of Pi in the chemosensitivity of osteosarcoma cells to other anticancer drugs, and we investigated the possible antitumor effects of treatments based on $\mathrm{Pi}$ in combination with either Taxol or 5-FU or doxorubicin in osteosarcoma U2OS cells. We showed that $\mathrm{Pi}$ increases the antiproliferative response to Taxol similarly to that caused by doxorubicin. In contrast, Pi did not potentiate the anticancer effects induced by 5-FU. These effects were paralleled by apoptosis induction and were cell cycle-dependent.

\section{Materials and methods}

Materials. All cell culture materials were from Gibco-Life Technologies (Gaithersburg, MD, USA). The anticancer drugs doxorubicin, Taxol (paclitaxel) and 5-FU were purchased from Sigma-Aldrich (St. Louis, MO, USA). Anti-tubulin antibodies were obtained from Oncogene-Calbiochem (La Jolla, CA, USA). Anti-procaspase-3, and anti-poly(ADP ribose) polymerase (PARP) antibodies were obtained from Upstate Biotechnology Inc. (Lake Placid, NY, USA). All other antibodies were obtained from Santa Cruz Biotechnology (San Diego, CA, USA).

Cell culture and treatments. The human osteosarcoma U2OS cell line was obtained from the American Type Culture Collection (Manassas, VA, USA). U2OS cells were grown in Dulbecco's modified Eagle's medium (DMEM) supplemented with $2 \mathrm{mM}$ glutamine, $100 \mathrm{U} / \mathrm{ml}$ penicillin, $100 \mu \mathrm{g} / \mathrm{ml}$ streptomycin and $10 \%$ fetal bovine serum (FBS) and cultured at $37^{\circ} \mathrm{C}$ in a $5 \% \mathrm{CO}_{2}$ humidified atmosphere. Unless noted, all experiments were conducted in the above medium which contained $1 \mathrm{mM}$ of $\mathrm{Pi}$, and the concentrations listed in the figures are final $\mathrm{Pi}$ medium concentrations. Added $\mathrm{Pi}$ was in the form of $\mathrm{NaPO}_{4}$, $\mathrm{pH} 7.4$, from Sigma-Aldrich (16-19). Doxorubicin was dissolved in $\mathrm{ddH}_{2} \mathrm{O}$ and stored at $4^{\circ} \mathrm{C}$; stock solutions of Taxol and 5-FU in $100 \%$ dimethylsulphoxide were maintained at $-20^{\circ} \mathrm{C}$ and thawed immediately before treatment of human tumor cells. Appropriate drug concentrations were made by dilution with fresh medium immediately before each experiment to final concentrations as indicated in the figures (20-22). Typically, subconfluent cells were split $\left(5 \times 10^{5} / 10 \mathrm{~cm}\right.$ plate $)$ and grown in
$10 \%$ serum containing medium. After $24 \mathrm{~h}$, the medium was removed, the cells were washed with PBS and incubated with $10 \%$ FBS fresh medium (time 0 ), supplemented or not with $\mathrm{Pi}$ and anticancer drugs, as single agents or in combination, and grown for the times and at concentrations indicated in the figures. Floating cells were recovered from culture medium by centrifugation, and adherent cells were harvested by trypsinization. Both floating and adherent cells were used in the experiments aimed to study expression of proteins involved in apoptosis and to perform FACS analysis (23).

Cell proliferation assay. Viable cells were determined by the 3-[4,5-dimethylthiazol-2-yl]-2,5-diphenyltetrazolium bromide (MTT) assay, as previously described $(18,24)$. Briefly, cells were seeded in 96-multi-well plates at the density of $5 \times 10^{3}$ cells/well. Cells were treated with Pi and drugs alone or in combination for up to $72 \mathrm{~h}$. Before harvesting, $100 \mu \mathrm{l}$ of MTT solution $(5 \mathrm{mg} / \mathrm{ml})$ was added to each well and incubated at $37^{\circ} \mathrm{C}$ for $3 \mathrm{~h}$, then the formazan product was solubilized by the addition of $100 \mu \mathrm{l} 0.04 \mathrm{~N} \mathrm{HCl}$ isopropanol. The optical density of each sample was determined by measuring the absorbance at 570 vs. $650 \mathrm{~nm}$ using an enzyme-linked immunosorbent assay reader (Molecular Device). Cell proliferation assays were performed at least three times (in replicates of 6 wells for each data point in each experiment). Data are presented as means \pm standard deviation for a representative experiment.

Evaluation of subG1 and cell cycle phases by flow cytometry. After drug treatment, cells were recovered as previously described in 'Cell culture and treatments', fixed by resuspension in $70 \%$ ice-cold methanol/PBS and incubated overnight at $4^{\circ} \mathrm{C}$. After fixing, samples were pelleted at $400 \mathrm{x}$ g for $5 \mathrm{~min}$, and pellets were washed once with ice-cold PBS and centrifuged for a further $5 \mathrm{~min}$. Pellets were resuspended in $0.5 \mathrm{ml}$ DNA staining solution [50 $\mu \mathrm{g} / \mathrm{ml}$ of propidium iodide (PI) and $100 \mu \mathrm{g}$ RNase A in PBS], and incubated at $37^{\circ} \mathrm{C}$ for $1 \mathrm{~h}$ in the dark. Samples were transferred to 5-ml Falcon tubes and stored on ice until assayed. Flow cytometric analysis was performed using a FACSCalibur flow cytometer (Becton Dickinson, San Jose, CA, USA) interfaced with a Hewlett-Packard computer (model 310) for data analysis. For the evaluation of intracellular DNA content, at least 20,000 events for each point were analyzed, and regions were set up to acquire quantitative data of cells with fragmented DNA (subG1 or apoptotic events) compared with the events that fell into the normal G1, S, G2 regions $(23,25)$.

Flow cytometric analysis of apoptosis. Annexin V-FITC (fluorescein isothiocyanate) was used in conjunction with a vital dye, PI, to distinguish apoptotic (Annexin V-FITC-positive, PI-negative) from necrotic (Annexin V-FITC-positive, PI-positive) cells (26). Briefly, cells were incubated with Annexin V-FITC (MedSystems Diagnostics, Vienna, Austria) and PI (Sigma-Aldrich) in a binding buffer (10 mM HEPES, $\mathrm{pH} 7.4,150 \mathrm{mM} \mathrm{NaCl}, 5 \mathrm{mM} \mathrm{KCl}, 1 \mathrm{mM} \mathrm{MgCl} \mathrm{Mg}_{2}, 2.5 \mathrm{mM}$ $\mathrm{CaCl}_{2}$ ) for $10 \mathrm{~min}$ at room temperature, washed and resuspended in the same buffer. Analysis of apoptotic cells was performed by flow cytometry (FACScan, Becton Dickinson). For each sample, $2 \times 10^{4}$ events were acquired. Analysis was 

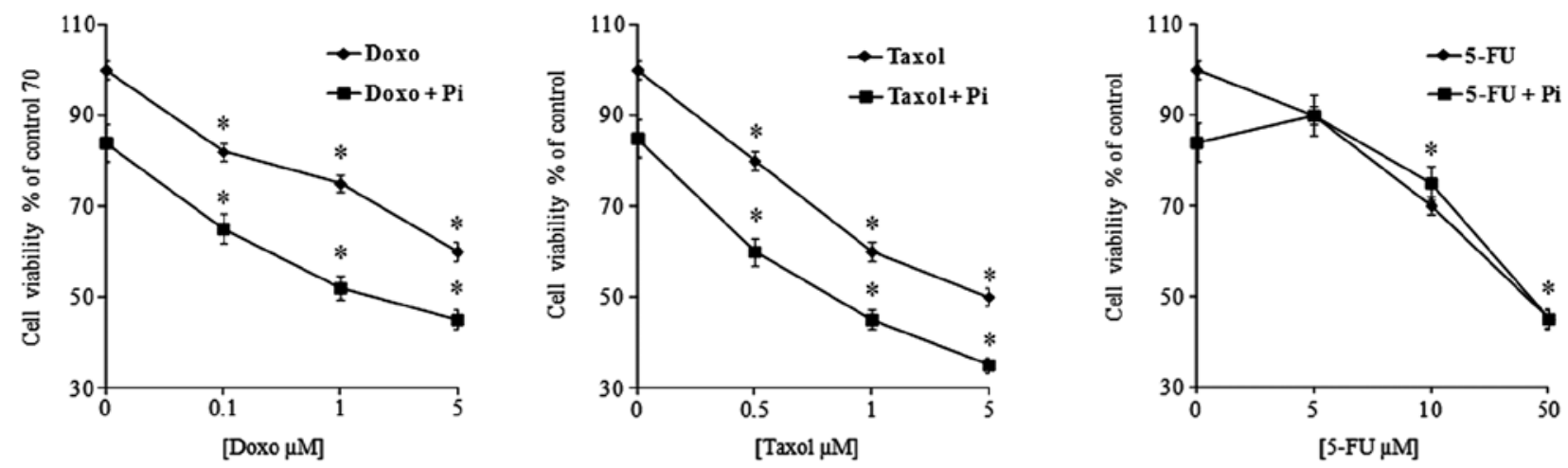

Figure 1. Effects of Pi on the cytotoxic effect induced by doxorubicin (Doxo), Taxol and 5-fluorouracil (5-FU). Dose-response experiments. U2OS cells were treated or not with increasing concentrations of doxorubicin, Taxol, 5-FU as single agents or in combination with $5 \mathrm{mM}$ Pi for $24 \mathrm{~h}$. The cell viability was then assessed by MTT assay. Data represent the average of three independent experiments. The means and SD are shown. ${ }^{*}<0.05$ vs. control untreated cells.

carried out by triplicate determination for at least three separate experiments

Preparation of cell lysates. Cell extracts were prepared as follows. Briefly, 3-5 volumes of RIPA buffer (PBS, 1\% NP-40, $0.5 \%$ sodium deoxycholate, $0.1 \%$ SDS) containing $10 \mu \mathrm{g} / \mathrm{ml}$ aprotinin, leupeptin, and $1 \mathrm{mM}$ phenylmethylsulfonyl fluoride (PMSF) were added to recovered cells. After incubation on ice for $1 \mathrm{~h}$, samples were centrifuged at 18,000 $\mathrm{x} \mathrm{g}$ in an Eppendorf microcentrifuge for $15 \mathrm{~min}$ at $4^{\circ} \mathrm{C}$ and the supernatant (SDS total extract) was recovered. Some aliquots were taken for protein quantification according to the Bradford method (27). Others were diluted in 4X Laemmli buffer, boiled and stored as samples for immunoblot analysis.

Immunodetection of proteins. Typically, we employed 20-40 $\mu \mathrm{g}$ of total extracts for immunoblotting. Proteins from cell preparations were separated by SDS-PAGE and transferred onto nitrocellulose sheets (Schleicher \& Schuell, Dassel, Germany) by a Mini Trans-Blot apparatus (Bio-Rad, Hercules, CA, USA). Secondary goat anti-rabbit or anti-mouse antibodies, conjugated with horseradish peroxidase (Bio-Rad), were used as a detection system (ECL) according to the manufacturer's instructions (Amersham Biosciences, UK).

Statistical analysis. Most of experiments were performed at least three times with replicate samples, except where otherwise indicated. Data are plotted as means \pm SD (standard deviation). The means were compared using analysis of variance (ANOVA) plus Bonferroni's t-test. A P-value of $<0.05$ was considered to indicate a statistically significant result. National Institutes of Health Image J 1.42Q (NIH, Bethesda, MD, USA) software was used for densitometric analysis.

\section{Results}

Pi enhances the cytotoxic effect induced by doxorubicin and Taxol, but not that induced by 5-FU in U2OS cells. To further investigate the antitumor action of $\mathrm{Pi}$ in osteosarcoma cells, we analyzed the potential antitumor effects of a combination of Pi and other commonly used chemotherapeutic agents. To this purpose, we treated osteosarcoma U2OS cells with varying concentrations of Taxol and 5-FU, in the presence or absence of $5 \mathrm{mM} \mathrm{Pi}$, a concentration covering the physiologic range in humans and in agreement with most of the published studies on Pi-triggered effects (15-19). Specific treatment conditions were examined encompassing exposure to no ( $0 \mathrm{mM}$, control), $0.5,1,5 \mu \mathrm{M}$ Taxol, and 5, 10, 50 $\mu \mathrm{M} 5-\mathrm{FU}$ in the presence or absence of $5 \mathrm{mM}$ Pi for $24 \mathrm{~h}$ (28-31). We also included in these experiments parallel cotreatments with $\mathrm{Pi}$ and doxorubicin $(0.1,1,5 \mu \mathrm{M})$ as previously reported (18). After the treatments, a conventional tetrazolium-based (MTT) assay was performed (Fig. 1). As expected, proliferation of U2OS cells was slightly inhibited in a dose-dependent manner by all three anticancer drugs used (32). Of note, we found that in all combinations, the presence of Pi strongly enhanced the antiproliferative effects of doxorubicin and Taxol (at a low dose of doxorubicin $0.1 \mu \mathrm{M}$ and Taxol $0.5 \mu \mathrm{M}$, the inhibition increased from 15 to $35 \%$ and from 20 to $40 \%$ in the presence of $\mathrm{Pi}$, respectively). The synergistic index (expressed as the ratio between the growth inhibition induced by the combination of the sum of the growth inhibitions caused by the single agents) was 1.4 and 1.3 for Doxo + Pi and Taxol + Pi, respectively. On the other hand, $\mathrm{Pi}$ combined with 5-FU did not induce any potentiation of the cytotoxicity caused by 5 -FU alone.

Moreover, we also studied the time-dependency of the growth inhibition caused by $\mathrm{Pi}+$ drug combinations. U2OS cells were exposed to no $(0 \mathrm{mM}$, control) or low doses of doxorubicin $(0.1 \mu \mathrm{M})$, or Taxol $(0.5 \mu \mathrm{M})$, or 5 -FU $(5 \mu \mathrm{M})$ in the presence or absence of $5 \mathrm{mM}$ Pi for 24, 48 and $72 \mathrm{~h}$ (Fig. 2). As expected, proliferation of U2OS cells was greatly decreased in a time-dependent manner by all three used agents. Notably, the growth inhibitory effects induced by Doxo $+\mathrm{Pi}$ and Taxol $+\mathrm{Pi}$ combinations were significantly higher than those caused by doxorubicin and Taxol alone, respectively (for doxorubicin, at $48 \mathrm{~h}$ the inhibition increased from 30 to $50 \%$ with an additive/ synergistic index of 1.1 and at $72 \mathrm{~h}$ from 40 to $65 \%$ with an additive/synergistic index of 1.1; for Taxol, at $48 \mathrm{~h}$ the inhibition increased from 44 to $61 \%$ with an additive/synergistic index of 1.1; at $72 \mathrm{~h}$ from 53 to $72 \%$ with an additive/synergistic index of 1.0). In contrast, no additive effects were noted with the $\mathrm{Pi}$ and 5-FU combination (Fig. 2). 
A

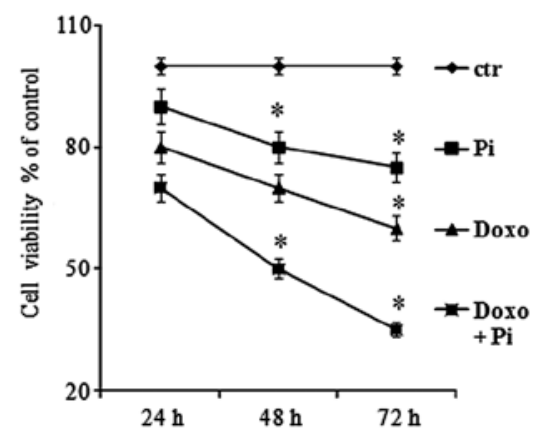

B

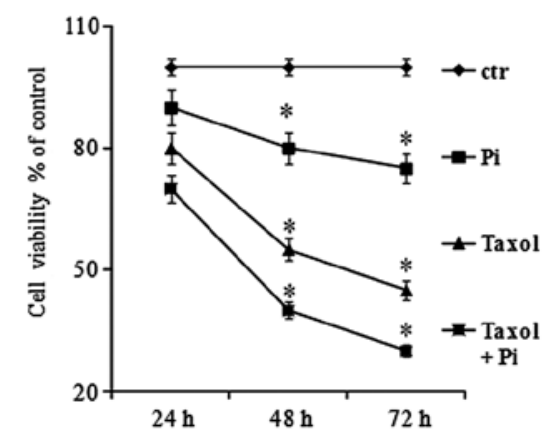

$\mathrm{C}$

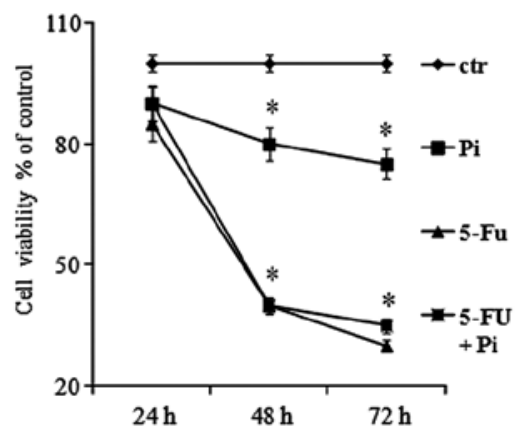

Figure 2. Effects of Pi on the cytotoxic effect induced by doxorubicin (Doxo), Taxol and 5-fluorouracil (5-FU) during a time course of $72 \mathrm{~h}$. U2OS cells were treated or not with $0.1 \mu \mathrm{M}$ doxorubicin (A), $0.5 \mu \mathrm{M}$ Taxol (B), $5 \mu \mathrm{M} 5-\mathrm{FU}$ (C) as single agents or in combination with $5 \mathrm{mM}$ Pi for 24,48 and $72 \mathrm{~h}$. The cell viabilitiy was then assessed by MTT assay. Data represent the average of three independent experiments. The means and SD are shown. ${ }^{*} \mathrm{P}<0.05$ vs. control untreated cells.

A No Drug
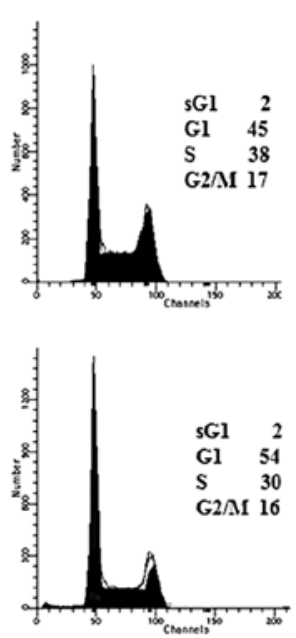

Doxo
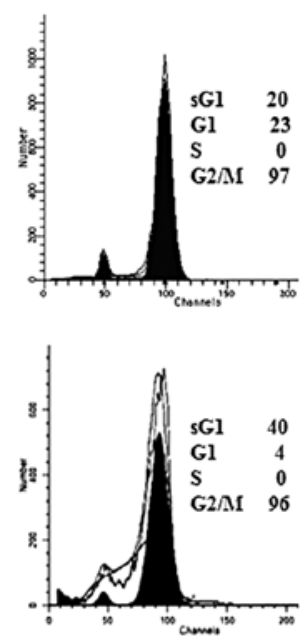

Taxol
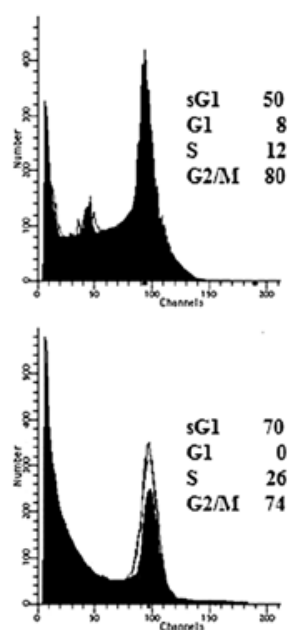

5-FU

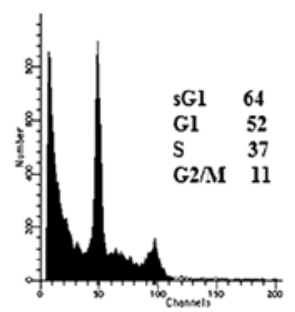

CTR

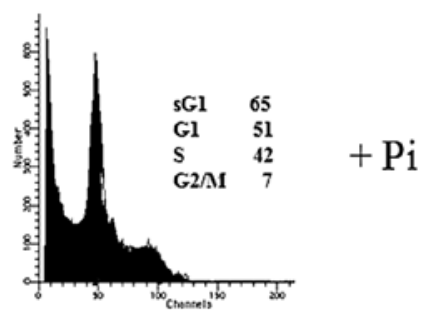

B

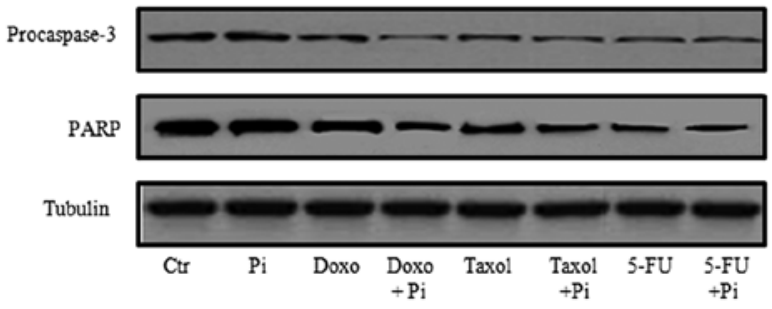

Figure 3. Effects of single-agent treatments of doxorubicin (Doxo), Taxol or 5-fluorouracil (5-FU), or their combinations with Pi on the cell cycle and apoptosis of U2OS cells. U2OS cells were treated or not with $0.1 \mu \mathrm{M}$ doxorubicin, $0.5 \mu \mathrm{M}$ Taxol, $5 \mu \mathrm{M}$ 5-FU as single agents or in combination with $5 \mathrm{mM}$ Pi for $48 \mathrm{~h}$. (A) Representative FACS histograms of propidium iodide-stained cells (20,000 events/sample) are shown. The percentage of cells in the hypoploid subG1 and of each cell-cycle phase is indicated. (B) Effects on pro-caspase-3 and PARP protein levels are shown. Cell extracts (30 $\mu \mathrm{g})$ from cells were subjected to SDS-PAGE and blotted with antibodies against the indicated proteins ( $\alpha$-tubulin was used as a standard for the equal loading of protein in the lanes). The image is representative of three immunoblot analyses from three different cellular preparations with similar results.

Pi potentiates the Taxol- and doxorubicin-induced cytotoxic effect in U2OS cells by inducing apoptosis. To gain further insight into the enhancement by Pi of doxorubicin- and Taxol-induced cytotoxicity, we performed western blotting and flow cytometry-based assays to study the cell cycle progression and apoptosis (Fig. 3). To this purpose, U2OS cells were exposed to no (0 mM, control), $0.1 \mu \mathrm{M}$ doxorubicin, $0.5 \mu \mathrm{M}$ Taxol, $5 \mu \mathrm{M}$
5-FU in the presence or absence of $5 \mathrm{mM}$ Pi for $48 \mathrm{~h}$ and FACS and western blot analysis were performed (Fig. 3).

As expected, Fig. 3A shows that Taxol- and doxorubicin-treated U2OS cells were highly accumulated in the G2/M phase with a concomitant decrease in the number of cells in the G1 and $\mathrm{S}$ phases of the cell cycle, whereas 5-FU caused the cells to accumulate in the $\mathrm{G} 1$ and $\mathrm{S}$ phases $(28,33)$. 
A

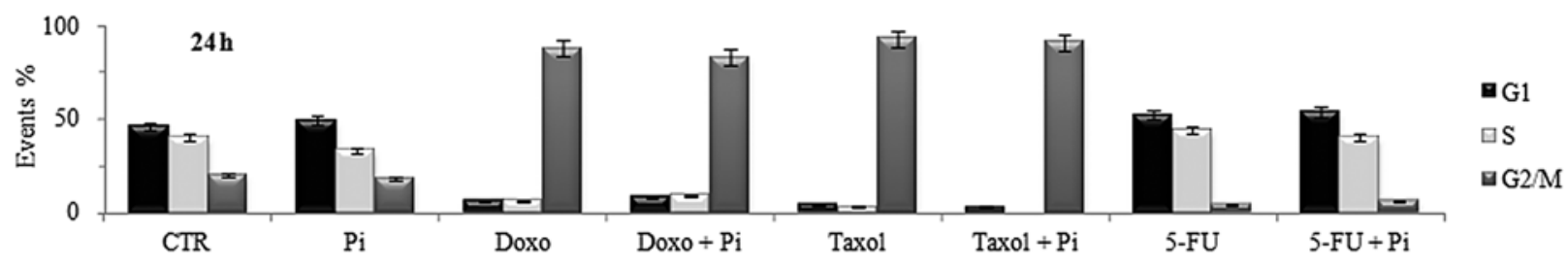

B

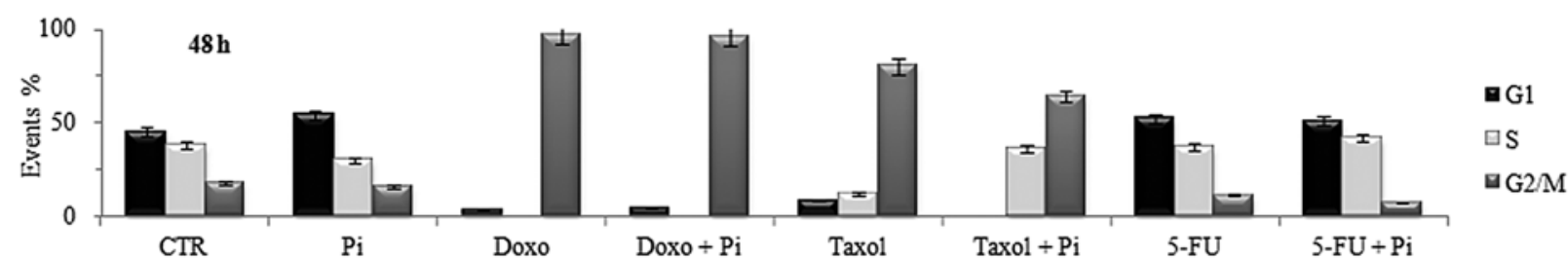

$\mathrm{C}$

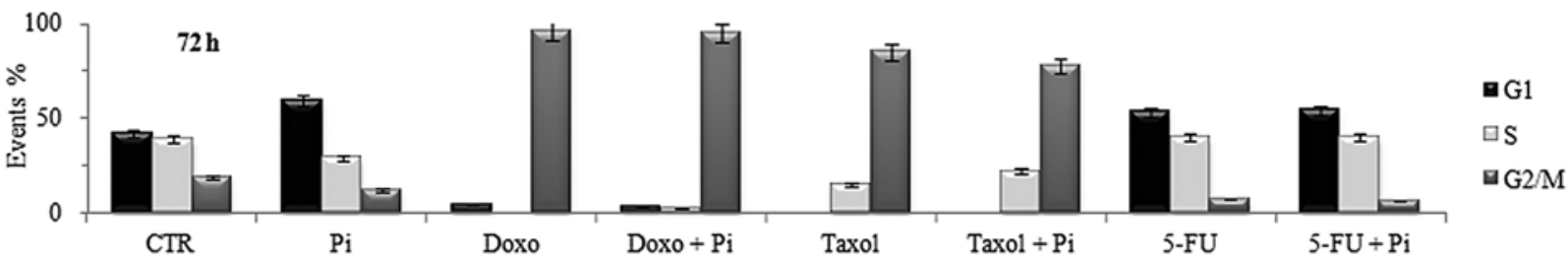

D

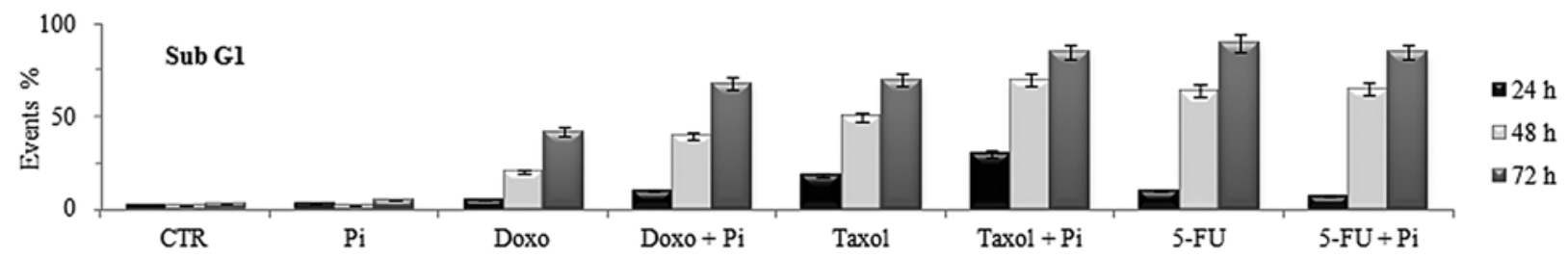

Figure 4. Effects of single-agent treatments of doxorubicin (Doxo), Taxol, 5-fluorouracil (5-FU) and their combinations with Pi during a time course of $72 \mathrm{~h}$ on the distribution of U2OS cells in subG1 and other cell cycle phases. U2OS cells were treated or not with $0.1 \mu \mathrm{M}$ doxorubicin, $0.5 \mu \mathrm{M}$ Taxol, $5 \mu \mathrm{M} 5$-FU as single agents or in combination with $5 \mathrm{mM}$ Pi for (A) 24, (B) 48 and (C) $72 \mathrm{~h}$. Then, FACS analysis of propidium iodide-stained cells was performed. Quantitative data indicating the percentage of hypoploid subG1 (D), G1, S and G2/M (A-C) phase cells from three independent experiments are shown. The means and SD are shown.

Table I. Study of apoptosis in the U2OS cell line.

\begin{tabular}{lcccc}
\hline $\begin{array}{l}\text { Treatment } \\
(48 \mathrm{~h})\end{array}$ & Necrosis & $\begin{array}{c}\text { Late } \\
\text { apoptosis }\end{array}$ & Alive & $\begin{array}{c}\text { Early } \\
\text { apoptosis }\end{array}$ \\
\hline Control & 0.15 & 0.4 & 99.25 & 0.2 \\
Pi & 0.17 & 0.65 & 98.88 & 0.3 \\
Doxo & 0.37 & $\mathbf{8 . 8 3}$ & 79.18 & $\mathbf{1 1 . 6 2}$ \\
Doxo + Pi & 0.51 & $\mathbf{2 5 . 7 9}$ & 61.38 & $\mathbf{1 1 . 3 2}$ \\
Taxol & 1.2 & $\mathbf{2 5 . 1}$ & 53.38 & $\mathbf{2 0 . 3 2}$ \\
Taxol + Pi & 1.81 & $\mathbf{3 6 . 1 9}$ & 33.73 & $\mathbf{2 8 . 2 7}$ \\
5-FU & 2.13 & 34.77 & 36.2 & 26.9 \\
5-FU + Pi & 2.3 & 36.91 & 37.1 & 23.69 \\
\hline
\end{tabular}

Bold values indicate significant changes. $\mathrm{Pi}$, inorganic phosphate; Doxo, doxorubicin; 5-FU, 5-fluorouracil.

Moreover, a subG1 cell population was noted in response to doxorubicin $(20 \%)$ and this was increased in response to
Taxol (50\%) and 5-FU (64\%). Notably, Fig. 3A shows also that treatment with Doxo $+\mathrm{Pi}$ and Taxol + Pi combinations induced a marked increase in the subG1 population when compared to treatment with the single agents (from 20 to $41 \%$ for Doxo + Pi vs. doxorubicin alone; from 50 to $70 \%$ for Taxol + Pi vs. Taxol alone), whereas the treatment with $\mathrm{Pi}$ alone did not cause any relevant change in the subG1 cell fraction. No increase in the subG1 population occurred following treatment with the 5-FU + Pi combination when compared to 5-FU treatment alone.

Accumulation of cells with a hypodiploid DNA content is consistent with cell death by apoptosis. This is further confirmed by examining the activation of the terminal caspase-3, executioner of apoptosis and cleavage of poly(ADP-ribose) polymerase, PARP, a known target for apoptosis-associated caspase cleavage $(18,29,30)$.

Fig. 3B shows a strong decrease in the uncleaved isoform of caspase- 3 in Doxo + Pi- and Taxol + Pi-treated cells suggesting an increase in its activity correlated with its fragmentation. Doxorubicin, Taxol and 5-FU alone induced similar effects on caspase-3 activation but to a less extent. Notably, no decrease in the procaspase-3 protein level was observed in U2OS cells 
treated with Pi alone. Finally, we evaluated the effects of the different treatments on the fragmentation of PARP, a substrate for caspase-3. The pattern of PARP processing paralleled that of caspase-3 cleavage (Fig. 3B).

In addition, to further prove the effects of the different treatments on apoptosis induction, we assessed apoptosis by FACS analysis after double labeling with Annexin V and PI (Table I). Overall, the data indicated that Pi potentiated the Taxol- and doxorubicin-induced antiproliferative effects (but not the 5-FU-induced ones) by inducing apoptosis of G2/Marrested U2OS cells.

To more effectively evaluate the effects of the combined treatments with $\mathrm{Pi}$ and the above drugs on cell cycle progression and apoptosis, we also analyzed the subG1 population and cell cycle distribution of U2OS cells during a time course (Fig. 4). Doxorubicin-induced G2/M accumulation was already evident at $24 \mathrm{~h}$ (>80\% cells in $\mathrm{G} 2)$ and increased at $48 \mathrm{~h}$ and was maintained up to $72 \mathrm{~h}$ ( $>90 \%$ cells in G2) (Fig. 4A-C). Noteworthy, Doxo + Pi combination induced a more time-dependent increase in the subG1 population $(10,41$ and $68 \%$ at 24,48 , $72 \mathrm{~h}$, respectively, compared to doxorubicin alone), whereas the treatment with $\mathrm{Pi}$ alone did not cause any relevant change in the subG1 cell fraction (Fig. 4D). Moreover, Taxol-induced $\mathrm{G} 2 / \mathrm{M}$ accumulation was already evident at $24 \mathrm{~h}$ ( $>90 \%$ cells in $\mathrm{G} 2 / \mathrm{M}$ ) and was maintained up to $72 \mathrm{~h}$ (Fig. 4A-C). Notably, Taxol + Pi combination induced a stronger time-dependent increase in subG1 cell population $(30,70$ and $85 \%$ at 24,48 , $72 \mathrm{~h}$, respectively, compared to Taxol alone) (Fig. 4D). In contrast, a large time-dependent increase in the subG1 population in response to 5-FU was evident, but no additive effect by Pi was noted (Fig. 4D).

\section{Discussion}

Due to its high metastatic potential and the frequent acquisition of chemotherapeutic resistance, the clinical outcome for osteosarcoma remains discouraging despite aggressive treatments. Thus, novel therapeutic approaches are urgently needed (3-5). Combination chemotherapy is receiving increased attention in order to identify compounds that may increase the therapeutic index of clinical anticancer drugs. In this regard, naturally occurring molecules with antitumor activity and with low toxicity to normal tissues have been suggested as possible candidates for investigation of their synergistic efficacy in combination with antineoplastic drugs.

Noteworthy, $\mathrm{Pi}$ is emerging as an important signaling molecule capable of modulating multiple cellular functions by altering signal transduction pathways, gene expression and protein abundance in many cell types (15). Previously, we provided evidence that $\mathrm{Pi}$ inhibits proliferation and aggressiveness of human osteosarcoma U2OS cells identifying adenylate cyclase, $\beta 3$ integrin, Rap1, ERK1/2 as proteins whose expression and function are relevantly affected in response to $\operatorname{Pi}(16,17)$.

In agreement with our previous findings, it has been reported that $\mathrm{L} / \mathrm{B} / \mathrm{K}$ ALP, alkalin phosphatase, whose main action is to locally increase the Pi levels in the extracellular environment, inhibits the aggressiveness and the metastatic ability of U2OS cells, by modulating the expression of genes involved in cell proliferation and adhesion (34). More recently, we described that in wild-type p53-containing osteosarcoma U2OS cells, and not in p53-null Saos and p53-mutant MG63 osteosarcoma cells, $\mathrm{Pi}$ is capable of inducing sensitization to doxorubicin in a p53-dependent manner (18). To the best of our knowledge, there have been no studies on the antitumor effect of inorganic phosphate on osteosarcoma cells, and our above studies are the first evidence.

In order to gain further insight into the antitumor action of $\mathrm{Pi}$ in osteosarcoma cells, we analyzed the possible antitumor effects of combined treatments with Pi and other commonly used chemotherapeutic agents compared to doxorubicin in osteosarcoma cells. The present study showed that osteosarcoma U2OS cells are significantly more sensitive to doxorubicin and Taxol when co-treated with Pi compared to the single use of these agents. In contrast, combined treatment of Pi with 5-FU did not have an additive antiproliferative effect compared to 5-FU alone.

The fact that inorganic phosphate, a simple 'naturally occurring molecule', combined with relevant chemotherapeutic agents can achieve this additive cytotoxic effect illustrates its potential for clinical applications. In addition, the fact that Pi enhances the antiproliferative effects of doxorubicin and Taxol in U2OS cells, but not those of 5-FU suggests that Pi does not act in a widespread way, but can have discrete effects on cell proliferation depending very likely on cell cycle phase(s) in which they occur. Doxorubicin, Taxol and 5-FU are widely used anticancer drugs belonging to different classes of chemotherapeutics (35).

Doxorubicin, classified as an anthracycline antiobiotic, is a DNA-damaging agent that generates DNA double-strand breaks (DNA DSBs) by inhibiting topoisomerase II $(36,37)$. Doxorubicin is a clinically relevant antitumor drug widely included in most chemotherapic treatment protocols for treating human osteosarcoma. It is well known that treatment of cells (including osteosarcoma cells) with doxorubicin leads to cell cycle arrest at the G2/M phase and eventually to apoptosis $(33,38,39)$. Taxol (paclitaxel) was isolated from the bark of the Pacific yew Taxus brevifolia and is one of the most powerful antitumor drugs (40). Taxol acts as an anticancer agent by targeting microtubules, promoting their assembly and stabilization. Treatment of cells (including osteosarcoma cells) with Taxol disrupts the formation of normal spindles at metaphase, leading to an arrest of cells in the G2/M phase of the cell cycle and eventually to apoptotic cell death $(28,29)$.

The antimetabolite and thymidylate synthase inhibitor 5-FU is a fluoropyrimidine-based compound, acting mainly in the $\mathrm{S}$ phase of the cell cycle to inhibit DNA synthesis (41). Through the enzymatic activity of uridine phosphorylase, orotate phosphoribosyltransferase and thymidine kinase, 5-FU is converted intracellularly to several active metabolites including fluoro(deoxy)uridine monophosphates [F(d)UMP], all of which interfere with RNA and DNA homeostasis to induce arrest of cells in the G1/S phase of the cell cycle and eventually apoptotic cell death $(26,31)$.

Notably, we found that Pi augmented the cytotoxic effect and showed a synergistic induction of apoptosis in osteosarcoma U2OS cells when combined with either doxorubicin or Taxol 'G2/M blocking' agents, whereas no additive antiproliferative effects were noted in combined treatments with $\mathrm{Pi}$ and 'G1/S blocking' 5-FU agent. The molecular mechanisms 
underlying the Pi-mediated chemosensitivity of osteosarcoma cells to anticancer drugs are just beginning to be understood and we do know that further studies and more exhaustive experiments are warranted.

Previously, we provided evidence that Pi inhibits cell cycle progression of U2OS cells, without the occurrence of apoptosis, with a G1 cell accumulation and S phase decrease (16). Moreover, we also described that the enhancement of doxorubicin-induced cytotoxicity by $\mathrm{Pi}$ occurs via $\mathrm{p} 53$-dependent apoptosis and through a mechanism involving ERK1/2 downregulation (18). A possible role of p53 and/or ERK1/2 in the enhancement of Taxol-induced cytotoxicity by $\mathrm{Pi}$ in U2OS is also under investigation by us, and experiments aimed to investigate the relationship between the combined treatments with Pi and chemotherapeutic drugs and their sequence are also planned. Irrespective of the mechanism(s), we report that $\mathrm{Pi}$ acts as a potent enhancer of doxorubicin- and Taxol-induced cytotoxicity in osteosarcoma cells.

Combination chemotherapy has received increased attention in order to identify compounds that may increase the therapeutic index of clinical anticancer drugs. Pi has been suggested as an attractive candidate to be investigated. In our study, Pi was found to have a positive pharmacological interaction even along with low doses of doxorubicin $(0.1 \mu \mathrm{M})$ and Taxol $(0.5 \mu \mathrm{M})$ that are expected to be more tolerable and associated with minimal undesired side effects in patients, thus increasing the potential clinical relevance of our data. New drug delivery systems have been developed that incorporate anticancer drugs into calcium phosphate cement (CPC) to maintain high concentrations of anticancer drugs at the local bone site (42). Of note, inorganic phosphate release and its bone retention from CPC is predicted to occur, thus affecting $\mathrm{Pi}$ concentrations locally.

Collectively, our data support the evidence of Pi as a signaling molecule and indicate that $\mathrm{Pi}$ may act as a potent enhancer of anticancer drug-induced cytotoxicity in osteosarcoma cells, suggesting that targeting Pi levels may contribute to the development of novel modalities for therapeutic intervention in osteosarcoma.

\section{Acknowledgements}

This study was supported by the Italian Minister for Research (contract grant sponsor, PRIN 2009).

\section{References}

1. Chou AJ, Geller DS and Gorlick R: Therapy for osteosarcoma: where do we go from here? Paediatr Drugs 10: 315-327, 2008.

2. Dai X, Ma W, He X and Jha RK: Review of therapeutic strategies for osteosarcoma, chondrosarcoma, and Ewing's sarcoma. Med Sci Monit 17: 177-190, 2011.

3. Kim SY and Helman LJ: Strategies to explore new approaches in the investigation and treatment of osteosarcoma. Cancer Treat Res 152: 517-528, 2009.

4. Hattinger CM, Pasello M, Ferrari S, Picci P and Serra M: Emerging drugs for high-grade osteosarcoma. Expert Opin Emerg Drugs 15: 615-634, 2010.

5. Gutierrez ME, Kummar S and Giaccone G: Next generation oncology drug development: opportunities and challenges. Nat Rev Clin Oncol 6: 259-265, 2009.

6. Raina K and Agarwal R: Combinatorial strategies for cancer eradication by silibinin and cytotoxic agents: efficacy and mechanisms. Acta Pharmacol Sin 28: 1466-1475, 2007.
7. Szekeres T, Saiko P, Fritzer-Szekeres M, Djavan B and Jäger W: Chemopreventive effects of resveratrol and resveratrol derivatives. Ann NY Acad Sci 1215: 89-95, 2011.

8. Tian L, Yin D, Ren Y, Gong C, Chen A and Guo FJ: Plumbagin induces apoptosis via the $\mathrm{p} 53$ pathway and generation of reactive oxygen species in human osteosarcoma cells. Mol Med Rep 5: 126-132, 2012.

9. Wu CL, Huang AC, Yang JS, et al: Benzyl isothiocyanate (BITC) and phenethyl isothiocyanate (PEITC)-mediated generation of reactive oxygen species causes cell cycle arrest and induces apoptosis via activation of caspase-3, mitochondria dysfunction and nitric oxide (NO) in human osteogenic sarcoma U-2 OS cells. J Orthop Res 29: 1199-1209, 2011.

10. Maran A, Shogren KL, Benedikt M, Sarkar G, Turner RT and Yaszemski MJ: 2-Methoxyestradiol-induced cell death in osteosarcoma cells is preceded by cell cycle arrest. J Cell Biochem 104: 1937-1945, 2008.

11. Chen JT, Fong YC, Li TM, et al: DDTD, an isoflavone derivative, induces cell apoptosis through the reactive oxygen species/ apoptosis signal-regulating kinase 1 pathway in human osteosarcoma cells. Eur J Pharmacol 597: 19-26, 2008.

12. Yoshiko Y, Candeliere GA, Maeda N and Aubin JE: Osteoblast autonomous Pi regulation via Pit1 plays a role in bone mineralization. Mol Cell Biol 27: 4465-4474, 2007.

13. Takeda E, Taketani Y, Sawada N, Sato T and Yamamoto H: The regulation and function of phosphate in the human body. Biofactors 21: 345-355, 2004.

14. Prié D, Beck L, Urena P and Friedlander G: Recent findings in phosphate homeostasis. Curr Opin Nephrol Hypertens 14: 318-324, 2005.

15. Khoshniat S, Bourgine A, Julien M, Weiss P, Guicheux J and Beck L: The emergence of phosphate as a specific signaling molecule in bone and other cell types in mammals. Cell Mol Life Sci 68: 205-218, 2011.

16. Naviglio S, Spina A, Chiosi E, et al: Inorganic phosphate inhibits grow th of human osteosarcoma U2OS cells via adenylate cyclase/ cAMP pathway. J Cell Biochem 98: 1584-1596, 2006.

17. Naviglio S, Di Gesto D, Borrelli V, et al: Novel molecular mechanisms by inorganic phosphate in osteosarcoma U2OS cells. Front Biosci 3: 1249-1258, 2011.

18. Spina A, Sorvillo L, Di Maiolo F, et al: Inorganic phosphate enhances sensitivity of human osteosarcoma U2OS cells to doxorubicin via a p53-dependent pathway. J Cell Physiol 228: 198-206, 2013.

19. Camalier CE, Young MR, Bobe G, Perella CM, Colburn NH and Beck GR Jr: Elevated phosphate activates N-ras and promotes cell transformation and skin tumorigenesis. Cancer Prev Res 3: 359-370, 2010.

20. Zou J, Gan M, Mao N, Zhu X, Shi Q and Yang H: Sensitization of osteosarcoma cell line SaOS-2 to chemotherapy by downregulating survivin. Arch Med Res 41: 162-169, 2010.

21. Olijslagers SJ, Zhang YH, Backendorf $\mathrm{C}$ and Noteborn $\mathrm{MH}$ : Additive cytotoxic effect of apoptin and chemotherapeutic agents paclitaxel and etoposide on human tumour cells. Basic Clin Pharmacol Toxicol 100: 127-131, 2007.

22. Mao FJ, Sidorova JM, Lauper JM, Emond MJ and Monnat RJ: The human WRN and BLM RecQ helicases differentially regulate cell proliferation and survival after chemotherapeutic DNA damage. Cancer Res 70: 6548-6555, 2010.

23. Naviglio S, Di Gesto D, Romano M, et al: Leptin enhances growth inhibition by cAMP elevating agents through apoptosis of MDA-MB-231 breast cancer cells. Cancer Biol Ther 8: 1183-1190, 2009.

24. Naviglio S, Di Gesto D, Illiano F, et al: Leptin potentiates antiproliferative action of cAMP elevation via protein kinase $\mathrm{A}$ down-regulation in breast cancer cells. J Cell Physiol 225: 801-809, 2010.

25. Naviglio S, Mattecucci C, Matoskova B, et al: UBPY: a growthregulated human ubiquitin isopeptidase. EMBO J 17: 3241-3250, 1998.

26. Lamberti M, Porto S, Marra M, et al: 5-Fluorouracil induces apoptosis in rat cardiocytes through intracellular oxidative stress. J Exp Clin Cancer Res 31: 60-68, 2012.

27. Bradford MM: A rapid and sensitive method for the quantification of microgram quantities of protein utilizing the principle of protein dye binding. Anal Biochem 72: 248-254, 1976.

28. Lu KH, Lue KH, Chou MC and Chung JG: Paclitaxel induces apoptosis via caspase-3 activation in human osteogenic sarcoma cells (U-2 OS). J Orthop Res 23: 988-994, 2005. 
29. Zhu JJ, Li FB, Zhou JM, Liu ZC, Zhu XF and Liao WM: The tumor suppressor p33ING1b enhances taxol-induced apoptosis by p53-dependent pathway in human osteosarcoma U2OS cells. Cancer Biol Ther 4: 39-47, 2005.

30. Song B, Wang Y, Xi Y, et al: Mechanism of chemoresistance mediated by miR-140 in human osteosarcoma and colon cancer cells. Oncogene 28: 4065-4074, 2009.

31. Im YS, Shin HK, Kim HR, et al: Enhanced cytotoxicity of 5-FU by bFGF through up-regulation of uridine phosphorylase 1 . Mol Cells 28: 119-124, 2009.

32. Koto K, Murata H, Kimura S, et al: Zoledronic acid inhibits proliferation of human fibrosarcoma cells with induction of apoptosis, and shows combined effects with other anticancer agents. Oncol Rep 24: 233-239, 2010.

33. Varmeh S and Manfredi JJ: Overexpression of the dual specificity phosphatase, Cdc25C, confers sensitivity on tumor cells to doxorubicin-induced cell death. Mol Cancer Ther 7: 3789-3799, 2008.

34. Zucchini C, Bianchini M, Valvassori L, et al: Identification of candidate genes involved in the reversal of malignant phenotype of osteosarcoma cells transfected with the liver/bone/kidney alkaline phosphatase gene. Bone 34: 672-679, 2004.

35. Agner J, Falck J, Lukas J and Bartek J: Differential impact of diverse anticancer chemotherapeutics on the Cdc25A-degradation checkpoint pathway. Exp Cell Res 302: 162-169, 2005.

36. Gewirtz DA: A critical evaluation of the mechanisms of action proposed for the antitumor effects of the anthracycline antibiotics adriamycin and daunorubicin. Biochem Pharmacol 57: 727-741, 1999.
37. Tentner AR,Lee MJ, Ostheimer GJ, Samson LD,LauffenburgerDA and Yaffe MB: Combined experimental and computational analysis of DNA damage signaling reveals context-dependent roles for Erk in apoptosis and G1/S arrest after genotoxic stress. Mol Syst Biol 31: 1-18, 2012.

38. Yuan XW, Zhu XF, Huang XF, et al: Interferon-alpha enhances sensitivity of human osteosarcoma U2OS cells to doxorubicin by p53-dependent apoptosis. Acta Pharmacol Sin 28: 1835-1841, 2007.

39. Chiosi E, Spina A, Sorrentino A, et al: Change in TNF-alpha receptor expression is a relevant event in doxorubicin-induced H9c2 cardiomyocyte cell death. J Interferon Cytokine Res 27: 589-597, 2007.

40. Zhao P and Astruc D: Docetaxel nanotechnology in anticancer therapy. Chem Med Chem 7: 952-972, 2012.

41. Longley DB, Harkin DP and Johnston PG: 5-fluorouracil: mechanisms of action and clinical strategies. Nat Rev Cancer 3: 330-338, 2003

42. Lopez-Heredia MA, Kamphuis GJ, Thüne PC, Öner FC, Jansen JA and Walboomers XF: An injectable calcium phosphate cement for the local delivery of paclitaxel to bone. Biomaterials 32: 5411-5416, 2011. 